\title{
The effectiveness of SPARX, a computerised self help intervention for adolescents seeking help for depression: randomised controlled non-inferiority trial
}

\author{
(c) $(1)(8)$ OPEN ACCESS
}

\author{
Sally N Merry associate professor ${ }^{1}$, Karolina Stasiak research fellow ${ }^{1}$, Matthew Shepherd lecturer ${ }^{2}$, \\ Chris Frampton associate professor of biostatistics ${ }^{3}$, Theresa Fleming research fellow ${ }^{1}$, Mathijs $\mathrm{F}$ \\ G Lucassen research fellow
}

'Department of Psychological Medicine, Faculty of Medical and Health Sciences, University of Auckland, Private Bag 92019, Auckland 1142, New Zealand; ${ }^{2}$ School of Counselling, Human Services and Social Work, Faculty of Education, University of Auckland; ${ }^{3}$ Department of Psychological Medicine, Christchurch School of Medicine and Health Sciences, University of Otago, New Zealand

\begin{abstract}
Objective To evaluate whether a new computerised cognitive behavioural therapy intervention (SPARX, Smart, Positive, Active, Realistic, X-factor thoughts) could reduce depressive symptoms in help seeking adolescents as much or more than treatment as usual.

Design Multicentre randomised controlled non-inferiority trial.

Setting 24 primary healthcare sites in New Zealand (youth clinics, general practices, and school based counselling services).

Participants 187 adolescents aged 12-19, seeking help for depressive symptoms, with no major risk of self harm and deemed in need of treatment by their primary healthcare clinicians: 94 were allocated to SPARX and 93 to treatment as usual.

Interventions Computerised cognitive behavioural therapy (SPARX) comprising seven modules delivered over a period of between four and seven weeks, versus treatment as usual comprising primarily face to face counselling delivered by trained counsellors and clinical psychologists.

Outcomes The primary outcome was the change in score on the children's depression rating scale-revised. Secondary outcomes included response and remission on the children's depression rating scale-revised, change scores on the Reynolds adolescent depression scale-second edition, the mood and feelings questionnaire, the Kazdin hopelessness scale for children, the Spence children's anxiety scale, the paediatric quality of life enjoyment and satisfaction questionnaire, and overall satisfaction with treatment ratings.

Results 94 participants were allocated to SPARX (mean age 15.6 years, $62.8 \%$ female) and 93 to treatment as usual (mean age 15.6 years, $68.8 \%$ female). 170 adolescents $(91 \%$, SPARX $n=85$, treatment as usual $\mathrm{n}=85)$ were assessed after intervention and $168(90 \%$, SPARX $n=83$, treatment as usual $n=85$ ) were assessed at the three month follow-up point. Per protocol analyses $(n=143)$ showed that SPARX was not inferior
\end{abstract}

to treatment as usual. Post-intervention, there was a mean reduction of 10.32 in SPARX and 7.59 in treatment as usual in raw scores on the children's depression rating scale-revised (between group difference $2.73,95 \%$ confidence interval -0.31 to $5.77 ; \mathrm{P}=0.079$ ). Remission rates were significantly higher in the SPARX $\operatorname{arm}(n=31,43.7 \%)$ than in the treatment as usual arm ( $n=19,26.4 \%$ ) (difference $17.3 \%, 95 \%$ confidence interval $1.6 \%$ to $31.8 \% ; P=0.030$ ) and response rates did not differ significantly between the SPARX arm $(66.2 \%, n=47)$ and treatment as usual arm (58.3\%, $n=42$ ) (difference $7.9 \%,-7.9 \%$ to $24 \%$; $P=0.332$ ). All secondary measures supported non-inferiority. Intention to treat analyses confirmed these findings. Improvements were maintained at follow-up. The frequency of adverse events classified as "possibly" or "probably" related to the intervention did not differ between groups (SPARX $n=11$; treatment as usual $n=11$ ).

Conclusions SPARX is a potential alternative to usual care for adolescents presenting with depressive symptoms in primary care settings and could be used to address some of the unmet demand for treatment.

Trial registration Australian New Zealand Clinical Trials ACTRN12609000249257.

\section{Introduction}

Up to a quarter of young people will have experienced a depressive disorder by the age of $19,{ }^{1}$ which is a major cause of disability. ${ }^{2}$ Effective therapies exist, ${ }^{3}$ with cognitive behavioural therapy recommended as the preferred treatment for mild to moderate depressive disorder. ${ }^{4}$ However, fewer than a fifth of young people with depressive disorder receive treatment, ${ }^{56}$ partly because of shortages in the workforce ${ }^{7}$ and partly because young people may be reluctant to seek traditional help. ${ }^{8}$ Computerised therapy offers a potential way forward and may appeal to today's "digital natives." 9 The cost of 
computerised therapy is likely to be substantially lower than traditional therapy and can increase access to treatment. ${ }^{10}$

Computerised cognitive behavioural therapy is effective for depressed adults, ${ }^{11}$ although adherence to treatment can be problematic. ${ }^{12}$ Recent reviews identified only eight studies on four computerised interventions for depression in children and adolescents. ${ }^{13}{ }^{14}$ None was a randomised controlled trial of the effectiveness of the intervention as a treatment for depression, but both reviews concluded that this approach is promising. We have carried out two small studies of computerised interventions for depressive symptoms; one showed a significant effect compared with placebo ${ }^{15}$ and the other was significantly more effective than a waitlist control. ${ }^{16} \mathrm{~A}$ small open trial carried out by another group showed encouraging results. ${ }^{17}$ The other papers identified were on depression prevention studies.

We designed a computerised cognitive behavioural therapy intervention, SPARX (Smart, Positive, Active, Realistic,

$\mathrm{X}$-factor thoughts), for adolescents seeking help for depression and tested its effectiveness compared with treatment as usual. We specifically aimed to design an intervention that would have wide appeal to young people in New Zealand and elsewhere and that would be enjoyable, thus resulting in good adherence rates. If computerised cognitive behavioural therapy can reduce symptoms and is at least as effective as treatment as usual it would be worth disseminating, because of the associated lower cost and the potential for reaching young people who currently do not access help.

\section{Methods}

Our primary hypothesis was that SPARX would reduce depressive symptoms as much or more than treatment as usual as measured on the children's depression rating scale-revised ${ }^{18}$ immediately after treatment compared with baseline. Our secondary hypotheses were that SPARX would lead to improvements as much or more than treatment as usual on self rated measures of depression, anxiety and hopelessness, and quality of life and on a clinician measure of overall improvement; and the change seen at the end of treatment would be maintained at follow-up (three months after completing the intervention).

\section{Trial design}

We carried out a randomised controlled non-inferiority trial to compare SPARX with treatment as usual. Outcome data were collected at baseline, post-intervention (about two months after the start of the intervention), and at follow-up three months later (five months after the start of the intervention). Safety data were collected at all time points, with an extra, brief check one month after the start of intervention. Satisfaction with the intervention was rated post-intervention and at the three month follow-up. The only change to study methods after the trial started was a change in sample size.

\section{Participants}

Young people seeking help for symptoms of depression, who met the inclusion criteria, were recruited sequentially through youth clinics (clinics specialising in providing primary healthcare to adolescents and young adults), general practices, and school based counselling services (in New Zealand, school guidance counsellors are trained to provide counselling for mental health problems and are often the first point of contact for adolescents with difficulties) in seven provincial and urban locations in New Zealand. We have labelled both healthcare professionals and school guidance counsellors as "clinicians." We asked clinicians to use the score of 10-19 on the patient health questionnaire-9 (the depression scale of the patient health questionnaire), ${ }^{19}$ as a guide to ensure that young people had clinically significant depression. However, we were inclusive and, if young people reported that the depression was troubling enough to need intervention and clinicians agreed with this assessment, we accepted them. Participants were enrolled in the trial according to the inclusion and exclusion criteria in box 1 .

\section{Interventions}

SPARX is an interactive fantasy game designed to deliver cognitive behavioural therapy for the treatment of clinically significant depression. It utilises both first person instruction and a three dimensional interactive game in which the young person chooses an avatar and undertakes a series of challenges to restore the balance in a fantasy world dominated by GNATs (Gloomy Negative Automatic Thoughts). The program is delivered on CD-ROM and has been designed to run on PCs with reasonably low specifications $(3 \mathrm{GHz}$ or faster Intel Pentium or equivalent processor, 512M RAM and 500MB hard disc space). The game consists of seven modules (levels), completed sequentially. Box 2 describes the content covered in each module. At the beginning and end of each module, the user interacts in the first person with a "guide," who puts the game into context, provides education, gauges mood, and sets and monitors real-life challenges, equivalent to homework. Young people who are not improving are prompted to seek help from their referring clinicians. SPARX is supplemented by a paper notebook with summaries of each module and spaces to add comments about the challenges completed (a trailer of SPARX is available at www.sparx.org.nz).

The control group was allocated to treatment as usual. At youth clinics and school based counselling services, treatment as usual was anticipated to be face to face therapy provided by trained counsellors or psychologists. At general practices, treatment as usual was likely to comprise assessment and treatment by general practitioners with psychological treatment where indicated and available. We collected data on the number and duration of sessions and type of therapy.

\section{Outcome measures}

\section{Primary outcome measure}

The children's depression rating scale-revised ${ }^{18}$ is an observer rated scale developed specifically for children but often used for adolescents. ${ }^{20}$ It has sound psychometric properties, ${ }^{21}$ is widely used in clinical research, ${ }^{21}$ and has been used to assess change in severity of depression in studies of treatment..$^{2022}$ The scale can be used as a screening and diagnostic tool and as a measure of severity of depression. It covers 17 symptom areas, which are rated on a seven point or five point scale, with the higher rating suggesting more severe difficulties. ${ }^{18}$ In our study population the Cronbach's $\alpha$ coefficient was 0.84 . We trained and supervised research assistants, who were blinded to the intervention, to carry out the ratings.

\section{Secondary outcome measures}

For our secondary outcome measures we chose the following self rated scales on the basis of psychometric properties and ease of use:

- The Reynolds adolescent depression scale-second edition ${ }^{23}$ is a 30 item self report measure developed to evaluate the severity of depressive symptoms in adolescents. ${ }^{20}$ Its reliability, validity, and normative data are well 


\title{
Inclusion criteria
}

Presented for treatment with symptoms indicative of mild to moderate depressive disorder

Aged 12 to 19 years on the date of consent

Provided written consent or, if under age 16 , written parental consent

Attended a clinical service or school based counselling service that was a study site

Achieved a minimum of one year of schooling in English

Had access to a computer to use SPARX

\section{Exclusion criteria}

A clinician assessed that the depression was too severe to make a self help resource a viable option

A clinician assessed the adolescent to be at high risk of self harm or suicide

Scored 7 on item 12 (morbid ideation) or 5 or higher on item 13 (suicidal ideation) on the children's depression rating scale-revised

Raw score was less than 30 on children's depression rating scale-revised

Intellectual disability or physical limitations precluded the use of the computer program

Had another major mental health disorder where the primary focus was not depression

Had had (in past three months) or was having treatment with cognitive behavioural therapy, interpersonal therapy, or antidepressants

\author{
Description of content and core skills covered in each module (level) of SPARX \\ Level 1-cave province: finding hope \\ Psychoeducation about depression and an introduction to the cognitive behavioural therapy model \\ Introducing GNATs (Gloomy Negative Automatic Thoughts) \\ Introducing "hope" (people recover from depression) \\ Relaxation: controlled breathing \\ Level 2-ice province: being active \\ Activity scheduling and behavioural activation \\ Relaxation: progressive muscle relaxation \\ Basic communication and interpersonal skills \\ Level 3-volcano province: dealing with emotions \\ Dealing with strong emotions: anger and hurt feelings \\ Interpersonal skills: assertiveness, listening, and negotiation \\ Level 4-mountain province: overcoming problems \\ Problem solving using STEPS: Say the problem, Think of solutions, Examine the pros and cons, Pick one and try it, See what happens \\ Cognitive restructuring-identifying SPARX: Smart, Positive, Active, Realistic, X-factor thoughts \\ Level 5-swamp province: recognising unhelpful thoughts \\ Cognitive restructuring—recognising different types of GNATs \\ Level 6-bridgeland province: challenging unhelpful thoughts \\ Cognitive restructuring-learning to challenge or "swap" negative thoughts for helpful ones \\ Interpersonal skills continued: negotiation skills \\ Level 7-canyon province: bringing it altogether \\ Recap of all skills \\ Mindfulness: tolerating distress \\ Relapse prevention: knowing when to ask for help
}

documented. ${ }^{20}$ Mean raw scores in the range $76-81$ fall into the mild clinical depression range. ${ }^{23}$ The scale has been validated for use in New Zealand adolescents. ${ }^{24}$ In our study population the Cronbach's $\alpha$ coefficient was 0.91 .

- The mood and feelings questionnaire ${ }^{25}$ is a brief (33 items) self administered measure designed to detect clinical depression in children and adolescents. In our study population the Cronbach's $\alpha$ coefficient was 0.91 .

- The paediatric quality of life enjoyment and satisfaction questionnaire $^{26}$ is a quality of life self report measure designed specifically for use in children and adolescents. It covers 15 areas and ratings are made on a five point scale from "very poor" to "very good." In our study population the Cronbach's $\alpha$ coefficient was 0.89 .
- The Spence children's anxiety scale ${ }^{27}$ is a well validated 46 item self report scale measuring child anxiety. In addition to a total score, it has six subscales, which measure specific types of anxiety. In our study population the Cronbach's $\alpha$ coefficient for the total score was 0.86 .

- The Kazdin hopelessness scale for children ${ }^{28}$ is a 17 item modification of the Beck hopelessness scale. ${ }^{28}$ It is self administered and has been widely used in studies of suicidality in youth. In our study population the Cronbach's $\alpha$ coefficient was 0.84 .

The clinicians also rated change on the improvement domain of the clinical global impression scale. ${ }^{29}$ It is a widely used single item scale requiring the assessor to rate how much the participant 
has changed relative to a baseline state (on a seven point scale from "very much improved" to "very much worse").

Participants completed a short questionnaire developed for the purpose of this study to assess satisfaction with and enjoyment of the allocated intervention.

\section{Sample size}

Using data from a pilot study by our group we calculated that about 300 participants per arm (600 total) would be required to power this non-inferiority study and allow some latitude for exploration of subgroup (sex, age, ethnicity, setting, and baseline depression) effects on efficacy. The pilot study included 34 participants with similar eligibility criteria, identical primary outcome measures, and the same timing of the primary outcome as participants in this trial. The standard deviation of the change on the children's depression rating scale-revised was 28. Using two tailed $95 \%$ confidence intervals, non-inferiority (not worse than 5.5 units inferior change on the children's depression rating scale-revised) would be identified with $80 \%$ power if about 600 participants were recruited. The children's depression rating scale-revised contains several categories-for example, "depressive disorder might be confirmed in a comprehensive diagnostic evaluation" and "depressive disorder is likely to be confirmed." The range of raw scores between these categories is 12-14. We argued that a difference of less than half a category was not likely to be clinically significant. When the recruitment rate was well below our anticipated targets, we extended the recruitment period and decided that adequately powered subgroup analyses would not be feasible. We recalculated the sample size for overall efficacy based on our accumulated data. This revised power calculation was undertaken as an interim summary of the overall variance of the change, not to test any of the study hypotheses, and therefore did not unblind us to treatment allocation. We estimated the variation in change on the children's depression rating scale-revised, based on the 55 participants (26 treatment as usual, 29 SPARX) who had baseline and post-intervention scores on that scale. The standard deviation of the change in scores based on this interim summary was 10.2 , considerably less than the original estimate. Using this estimate and the same variables above, the power calculation indicated a revised total sample size of 190 participants. This calculation allows for about $30 \%$ attrition from the intention to treat population; this $30 \%$ includes those potentially lost to follow-up or not adhering to the treatment protocol.

\section{Randomisation and sequence generation}

We randomly allocated participants to SPARX or treatment as usual in a 1:1 ratio, using a computer generated randomisation sequence prepared before any participants were randomised. Allocation was stratified by study site and arranged in permuted blocks of 4 .

Potential participants were informed about the study by their clinicians. A research assistant not involved in the participants' clinical care confirmed eligibility. To ensure allocation concealment, once eligibility had been confirmed, the participant was given an opaque sealed envelope containing the randomised allocation. The young person took this to a local investigator who opened the envelope, informed the young person of the allocation, and organised access to SPARX or treatment as usual. The assessment package and randomisation envelopes were stored off-site and were available only to the research assistants.

\section{Blinding}

It was not possible to blind the participants or treatment providers. However, trained research assistants, blinded to treatment allocation, carried out all assessments and administered the rating scales. Those analysing data were unaware of the treatment allocation. Research assistants, staff at treatment centres, and the participants were briefed to not discuss which treatment participants were receiving.

\section{Statistical analysis}

The primary analyses testing non-inferiority were undertaken on the per protocol population, with sensitivity analyses on the intention to treat population. ${ }^{30}$ Both sets of results are presented. The per protocol population included those who received SPARX as per protocol (defined as completing at least four out of seven modules) and met all other requirements of the protocol. We used a general linear mixed model analysis to compare changes in all metric outcomes between treatments, with the site/stratum as a random between participants factor, and the baseline level as a covariate. The results of these analyses are summarised as the mean changes ( $95 \%$ confidence intervals) for each group and the mean difference in these changes (95\% confidence intervals) between groups. We used $\chi^{2}$ tests to compare remission and response rates between randomised groups. Response was defined as a 30\% decrease in symptoms on the children's depression rating scale-revised ${ }^{18}$ after correcting for the non-zero minimum on the scale (by subtracting the scale minimum of 17 from each score), and remission was defined as a raw score on children's depression rating scale-revised of less than $30 .{ }^{18} \mathrm{We}$ used two sided $95 \%$ confidence intervals to summarise non-inferiority testing. As non-inferiority was confirmed to assist interpretation of results, we also show the $\mathrm{P}$ values generated from tests of superiority (as recommended previously ${ }^{30}$ ).

We undertook ancillary analyses on the primary outcome to confirm the consistency of any treatment effects across potentially prognostic subgroups. These subgroups were sex, age, ethnicity, setting, and baseline depression. These four subgroup analyses were undertaken using randomised treatment as a factor and the baseline level as a covariate (except when testing the subgroups defined by baseline depression level). The models also included the subgroup as a factor and the interaction between randomised treatment and subgroup to test the significance of any difference in treatment effects across the subgroups. Given the relatively small sample sizes of some of the subgroups, the power for testing these interactions is limited and these results should be considered exploratory only.

The safety data and adverse events were listed by treatment arm and included details of the event, its severity, and the likely relatedness to study treatment. A check for safety was carried out four weeks into the study, in line with usual clinical practice- that is, to carry out monthly check-ups for young people with a clinical presentation similar to that of our participants.

\section{Missing data}

For the intention to treat analyses, we estimated missing data using multiple imputation. This imputation process for each outcome utilised participant's sex, age, ethnicity, and setting and the relevant available assessments of the outcome. Ten imputations were undertaken for each imputation estimate and pooled results from these were used. 


\section{Results}

Participants were recruited at 24 sites, including 15 school based counselling services, seven youth clinics, and two general practices from May 2009 to July 2010. Follow-up was completed by December 2010. Recruitment stopped with 187 participants (of the anticipated 190) to fit in with school terms and to allow time to complete follow-up before the extended summer break (starts in December).

Of 213 young people assessed for eligibility to participate, 26 did not meet the inclusion criteria and 187 were randomised.

Overall, 170 participants $(91 \%)$ were assessed post-intervention and $168(90 \%)$ at the three month follow-up. Dropout rates were low (9\%) and comparable between the two groups (SPARX $9 / 94$, treatment as usual $8 / 93$, fig $1 \Downarrow$ ). The recruitment and randomisation strategy led to comparable treatment groups (table $1 \Downarrow$ ).

\section{Treatment as usual}

Data on the nature of treatment as usual were available for 83 (89\%) participants. Most received counselling ( $n=74,89.2 \%)$, some $(\mathrm{n}=11,13.3 \%)$ were waitlisted for active treatment, and two $(2.4 \%)$ were prescribed drugs. The mean number of treatment as usual sessions was 4.8 (median 4, range 1-20). Clinicians reported that, for $65(85.5 \%)$ of the young people receiving counselling, the sessions lasted at least 30 minutes, and for more than half $(n=44,57.9 \%)$ they were for 45 minutes or longer.

\section{Adherence rates}

Eighty out of 94 young people who were allocated to SPARX returned questionnaires reporting number of modules completed. Adherence rates for SPARX were good, with 69 (86\%) of participants allocated to SPARX completing at least four modules, $48(60 \%)$ completing all seven modules, and $50(62 \%)$ completing most or all of the homework challenges set. The most common reasons for non-completion were technical glitches, lack of time, lack of interest, not finding the resource helpful, or being physically unwell and unable to attend appointments.

\section{Outcomes and estimation}

\section{Per protocol analysis}

A per protocol analysis based on those who completed at least four of the SPARX modules and did not violate any protocol specifications was carried out on 143 participants and showed that SPARX was not inferior to treatment as usual on any measure. In the SPARX group the mean reduction on our primary outcome variable (children's depression rating scale-revised) was 10.32 (95\% confidence interval 8.15 to 12.48) and in the treatment as usual group 7.59 (5.43 to 9.75), with an advantage to SPARX of 2.73 (95\% confidence interval -0.31 to $5.77 ; \mathrm{P}=0.079)$. Compared with the treatment as usual groups, mean changes were significantly higher in the SPARX group on the Kazdin hopelessness scale for children, the mood and feelings questionnaire, and the Spence generalised anxiety subscale (see table 3 ). The clinical global impression-improvement response rate did not differ significantly between the groups $(\mathrm{P}=0.178)$.

\section{Intent to treat analysis}

Participants in the intention to treat analysis improved substantially, with a mean reduction on the children's depression rating scale-revised of 9.05 (95\% confidence interval 7.07 to
11.03) for SPARX and 7.45 (5.46 to 9.45) for treatment as usual, an advantage to SPARX of $1.60(-1.21$ to $4.41 ; \mathrm{P}=0.264)$ affirming non-inferiority, as the lower limit on this interval does not include -5.5 (fig $2 \Downarrow$ ). The effect size equates to Cohen's d (mean difference divided by the pooled standard deviation of the change) of 0.3. All secondary outcome measures improved, with in nearly all instances the SPARX group achieving mean changes better than those of the treatment as usual group (tables $2 \Downarrow$ and $3 \Downarrow)$.

\section{Response and remission rates}

In the per protocol analysis, remission rates on the primary outcome were significantly higher in the SPARX group $(n=31$, $43.7 \%)$ than in the treatment as usual group $(n=19,26.4 \%)$ : difference $17.3 \%$ (95\% confidence interval $1.6 \%$ to $31.8 \%$; $\mathrm{P}=0.030$ ). Rates of response to treatment did not differ significantly between the groups: $66.2 \%$ in the SPARX group $(\mathrm{n}=47)$ and $58.3 \%$ in the treatment as usual group $(\mathrm{n}=42)$ : difference $7.9 \%$ ( $-7.9 \%$ to $24 \%$; $\mathrm{P}=0.332)$.

In the intention to treat analysis, at post-intervention $59.6 \%$ $(n=56)$ of those receiving SPARX responded to treatment compared with $54.8 \%(\mathrm{n}=51)$ of those receiving treatment as usual: difference 4.7 ( -9.3 to 18.5$)$. Overall, $44.7 \%(n=42)$ of participants in the SPARX group recovered compared with $35.5 \%(n=33)$ in the treatment as usual group: difference 9.2 ( -4.8 to 22.7$)$. At follow-up, $81.9 \%(n=77)$ of those in the SPARX group and $74.2 \%(n=69)$ in the treatment as usual group had responded (difference $7.7,-4.2$ to 19.4$)$ and $58.5 \%(n=55)$ in the SPARX group and $52.7 \%(\mathrm{n}=49)$ in the treatment as usual group had recovered (difference $5.8,-8.3$ to 19.6). The differences between groups were not significant.

\section{Planned ancillary analyses}

\section{Sex, age, ethnicity, setting, and baseline depression}

As indicated by the interaction terms from the general linear mixed model analyses, the effectiveness of SPARX was not related to sex $(\mathrm{P}=0.962)$, age $(\mathrm{P}=0.277)$, ethnicity $(\mathrm{P}=0.428)$, or setting (school versus non-school, $\mathrm{P}=0.911$ ). However, compared with participants who were less depressed at baseline those who were more depressed had a significantly $(\mathrm{P}=0.038)$ greater reduction in depression scores in the SPARX group than in the treatment as usual group (table $4 \Downarrow$ ).

\section{Post hoc sensitivity analysis}

Unexpectedly, $13.3 \%$ of participants in the treatment as usual group were on a waiting list. The robustness of the findings was therefore tested by carrying out a post hoc analysis excluding young people on a waiting list. Non-inferiority of SPARX was confirmed by this analysis, which actually showed a greater improvement from SPARX than from treatment as usual on all measures. The mean changes, respectively, for the SPARX group and treatment as usual group on the children's depression rating scale-revised were -8.55 (95\% confidence interval -10.44 to -6.66$)$ and -6.41 ( -8.44 to $-4.38 ; \mathrm{P}=0.130)$; on the Reynolds adolescent depression scale-second edition were $-9.32(-11.76$ to -6.88 ) and -6.09 ( -8.71 to $-3.48 ; \mathrm{P}=0.076)$; on the moods and feelings questionnaire were $-8.55(-10.73$ to -6.36$)$ and $-5.23(-7.57$ to $-2.88 ; \mathrm{P}=0.042)$; and on the Spence children's anxiety scale were $-7.88(-10.04$ to -5.72$)$ and $-5.32(-7.63$ to $-3.01 ; \mathrm{P}=0.11$ ). 


\section{Safety and adverse events}

Forty nine adverse events were recorded during the study. These were similarly distributed between the intervention groups. Most were unrelated to the study (table $5 \Downarrow$ ).

Events classified as "possibly" or "probably" related to the study were rated by severity. One participant in the SPARX group and two in the treatment as usual group experienced mild adverse events and eight participants in each group experienced moderately severe events (for example, worsening of mood, increase in suicidal thinking). Two participants in the SPARX group and one in the treatment as usual group experienced serious events; all these were suicide attempts (one suicide attempt in the SPARX group occurred between randomisation and treatment and resulted in withdrawal from the study before intervention).

\section{Satisfaction with the program}

Satisfaction was assessed using a self report questionnaire designed for the study. The questionnaire consisted of Likert scales rating the features of the intervention, yes/no answers, and open ended items. The questionnaire about SPARX differed slightly from that about treatment as usual because it covered different aspects of the interventions. Both groups were asked whether they thought the intervention they had received (SPARX or treatment as usual) would appeal to other teenagers and whether they would recommend it to their friends. Eighty participants in the SPARX group and 71 in the treatment as usual group completed satisfaction questionnaires immediately after intervention; 76 (95.0\%) of participants in the SPARX group and $70(98.6 \%)$ in the treatment as usual group $(\mathrm{P}=0.371)$ believed that the type of support they received would appeal to other teenagers, and $64(80.5 \%)$ of participants in the SPARX group and $68(95.8 \%)$ in the treatment as usual group $(\mathrm{P}=0.005)$ would recommend the treatment to their friends.

Of those who completed SPARX and returned satisfaction questionnaires $(n=80), 53.2 \%(n=43)$ would have liked the sessions to stay the length they were (most reported taking 20 to 40 minutes to complete each module), $44.3 \%(n=35)$ wanted the sessions to be longer, and $61.5 \%(n=49)$ reported that they completed all or most of the set challenges ("homework"). Box 3 shows the most favourably rated features of both interventions.

\section{Discussion}

A computerised cognitive behavioural therapy program, SPARX, was an effective resource for help seeking adolescents with depression at primary healthcare sites. Use of the program resulted in a clinically significant reduction in depression, anxiety, and hopelessness and an improvement in quality of life. The results are more impressive when it is considered that SPARX was entirely a self help resource. The only contact with a clinician was at recruitment, and the only input from health professionals during the course of treatment was a brief phone call after a month. The intervention was at least as good as treatment as usual in primary healthcare sites in New Zealand (mainly comprising a high level of face to face counselling by trained and experienced staff) but would be cheaper and easier to disseminate. The treatment effects persisted to three months after the completion of the program. SPARX was more effective than treatment as usual for those who were most depressed at the start. Adherence rates were high. We did not find any differential effect across different ethnic groups in New Zealand or for males and females, although we were underpowered to detect differences for these subgroups. Most participants found SPARX useful, believed it would appeal to other teenagers, and would recommend it to their friends. Some evidence suggested that participants who completed at least four modules did significantly better than those who received treatment as usual. The response and remission rates for participants in the SPARX group (66\% and $44 \%$ respectively) compare favourably with other effective monotherapies, including antidepressants and cognitive behavioural therapy, ${ }^{31}$ whereas rates for treatment as usual were a little lower, possibly because some young people were on a waiting list. Although the magnitude of change on the children's depression rating scale-revised was lower than that of some of the drug trials, this is to be expected given our entry criteria. Studies of antidepressants typically include young people with moderate to severe depressive disorder, ${ }^{32}$ whereas we recruited those seeking help for depressive symptoms at a level that is typical of those who present at primary healthcare settings. ${ }^{33}$ The New Zealand guidelines recommend that for mild to moderate depression, young people should be offered psychological therapies as preferred treatment, whereas antidepressants are recommended for those who do not respond or for those presenting with more severe depression. ${ }^{34}$ This is probably the reason for the low rates of drug use in both groups.

\section{Strengths and weaknesses of the study}

This study was carried out according to CONSORT guidelines, in a range of primary healthcare sites. It was adequately powered to address the primary question of whether SPARX was inferior to treatment as usual; the study may, however, have been underpowered to detect superiority over treatment as usual. The participants were young people accessing services rather than those recruited by advertisement, and exclusion criteria were kept to a minimum to maximise generalisability. The primary outcome variable was assessed by research assistants blind to allocation. Care was taken to ensure allocation concealment. The treatment as usual group received the usual care delivered in these primary care sites. Our retention rates were excellent.

The study does have some weaknesses. Ideally we would have liked to have used more than one measure on interview, and originally we planned to include an interview assessment of diagnosis, which would also have allowed us to assess for comorbidity. This proved to be difficult logistically because of the extra time needed and the difficulties fitting this in with the services participating in the trial. It would have been easier in a research clinic but this would have compromised the generalisability to actual clinical service settings. We made the children's depression rating scale-revised our primary outcome variable in line with most studies of antidepressant drugs in this age group. The design of the study does not allow us to rule out spontaneous improvement in both groups. However, the mean duration of an episode of depressive disorder in this age group is six months ${ }^{35}$ and, in a comparable population to ours over $50 \%$ of young people presenting to primary care settings in the United Kingdom with similar levels of depression failed to recover within six months. ${ }^{33}$ The improvement associated with SPARX persisted for three months after the intervention was complete making a placebo effect unlikely. An earlier pilot study (by a member of our group) of a computerised intervention, The Journey, ${ }^{15}$ which informed the development of SPARX, showed that it was more effective than a placebo computerised intervention (Cohen's $\mathrm{d}=1.7$ ), and a companion study to this randomised controlled trial ${ }^{16}$ showed that SPARX was more effective than a waitlist situation in a group of young people excluded from mainstream education (Cohen's d=1.61). We do not have good data on adherence to treatment as usual. Clinicians often forgot to fill in our forms, and for some the number of sessions planned was unclear. Adherence to SPARX 


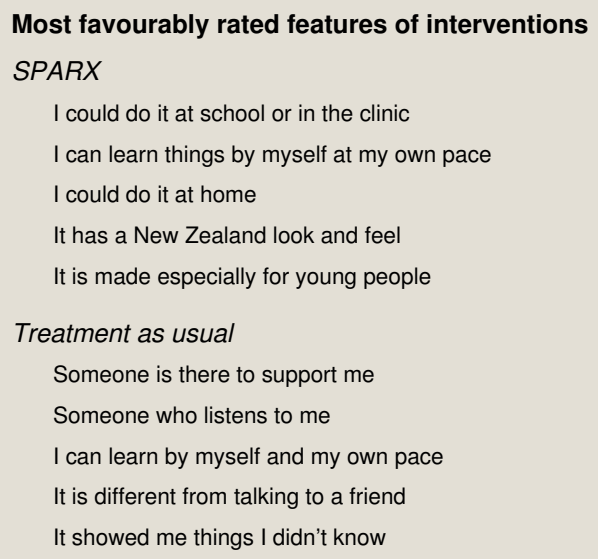

was based on participants' self report. Ideally, this would have been more robust, but attempts to collect these data on the computers at the different sites foundered as we experienced technical difficulties at some of the sites.

One of the main weaknesses of our study was the heterogeneity of our treatment as usual group. We considered it unethical to leave young people who had presented for help untreated. We did not have the resources to provide an alternative treatment, such as cognitive behavioural therapy or interpersonal therapy, the "gold standards." However, the question for the Ministry of Health (funder of the study) was whether or not SPARX was a reasonable treatment to be offered to those presenting in primary healthcare settings, including at school based counselling services, where resources are not sufficient to meet demand. The sensitivity analysis, in which we removed young people who were on the waiting list for treatment as usual, showed that SPARX was at least as effective as active treatment. SPARX may also have a place for the $80 \%$ of young people with depressive disorder who never receive treatment, ${ }^{5}$ some of whom seem likely to prefer an option to complete therapy in privacy and in their own time. The number of sites and clinicians and the work related stress in the various sites made it impossible to collect robust data on those that refused to take part in the study. However, clinicians reported that nearly all the young people they thought were suitable for the study agreed to take part. A few young people did not want to ask their parents for consent, and a small number did not want to be in the study because they did not like computers or because they specifically wanted to talk to someone. Overall, the rate of refusal was low and participants in this study are representative of those presenting for help for depressive symptoms.

\section{Comparison with other studies}

This is the third randomised controlled trial of a computerised intervention for depression in adolescents carried out by our group. The first, a small pilot study, ${ }^{15}$ showed a significant reduction in depressive symptoms compared with a computerised placebo program, and the second, also a small study, showed that SPARX was more effective than a waitlist situation in a group of young people excluded from mainstream education. ${ }^{16}$ A recent systematic review ${ }^{13}$ identified six studies of four computerised cognitive behavioural therapy interventions for depression in children and adolescents. Of these, two had no control group, two were studies of depression prevention in universal samples of school children, and the last was a randomised controlled trial comparing brief advice with motivational interviewing as a strategy to increase adherence to the computerised intervention. We updated the search with the same search terms used in the systematic review and identified two further studies. One, of depression prevention in a large universal sample of secondary school students, ${ }^{36}$ showed a modest effect only among males (Cohen's $d=0.43$ ). The second was a small pilot study in 15 adolescents, with encouraging but not definitive results. ${ }^{17}$ The format of SPARX led to good engagement with the resource, with $60 \%$ of participants completing all modules compared with $32 \%$ in the YouthMood project, ${ }^{36}$ although it should be noted that in our study the results were based on self report, whereas the data from the YouthMood project were collected automatically by the computerised program. In the study of Reach Out Central, the study authors were surprised that participants had accessed the program so infrequently, with on average boys completing 1.5 sessions and girls 1.6 sessions,${ }^{37}$ whereas $86 \%$ of our participants reported that they completed at least four modules. Participants allocated to SPARX also expressed high levels of satisfaction. Although $80 \%$ of young people in the SPARX group would recommend the program to their friends, significantly more young people receiving treatment as usual ( $96 \%$ ) would recommend that intervention to their friends. Some computerised interventions have been shown to be more effective than care delivered in general practice, ${ }^{38}$ but our participants were largely recruited from school based counselling services and youth clinics that provided psychological interventions by, for the most part, trained, experienced counsellors, so interventions were well above those usually available in a general practice setting. SPARX could be made widely available and could help to address the needs of those reluctant to seek help from a therapist, those on waiting lists, and those in areas where access to therapy is poor.

As data on computerised interventions in children and adolescents with depression were sparse, we have included a comparison of our findings in relation to the more extensive data on adults. A recent meta-analysis identified 22 randomised controlled trials of computerised therapy compared with another treatment or control condition ${ }^{11}$ of which six were for depressive disorder. To be included, the participants had to meet diagnostic criteria for major depressive disorder, panic disorder, social phobia, or generalised anxiety disorder. Seventeen of these studies used a waitlist situation as a comparison. Most ${ }^{11}$ of the studies used the internet to deliver cognitive behavioural therapy. In 12 of the studies, computerised cognitive behavioural therapy was supported by a therapist to a varying degree. The mean effect size (Hedges g) for a depressive disorder was 0.78 (based on six randomised controlled studies). Five randomised controlled trials compared computerised cognitive behavioural therapy with face to face treatment for depression or panic disorder and none showed significant differences between the 
treatments. There was no evidence of relapse in the 14 studies that reported follow-up data (median 26 weeks).

\section{Meaning of the study}

SPARX, a computerised self help intervention for adolescents with symptoms of depression, is at least as good as treatment as usual in primary healthcare settings in New Zealand and is a potentially useful treatment. It could be used as the first component in a stepped care approach to managing depression in this age group. As the participants in our study were young people recruited from clinical settings in a large number of sites and few eligible young people refused to participate, our sample is likely to be representative of young people seeking and receiving help for depression. Participants were similar to those presenting in primary care in a study carried out in the United Kingdom. ${ }^{33}$ In our study young people completed the resource independently, with minimal oversight, mostly on a computer in the facility where they had sought help, although some completed it at home. SPARX is an effective resource for adolescents with depressive symptoms. It is at least as good as treatment as usual, would be cheaper and easier to disseminate, and could be used to increase access to therapy. It could provide access to treatment for young people who may be reluctant to have more conventional therapy.

\section{Unanswered questions and future directions}

Although we had good adherence in the context of a study, adherence in day to day clinical practice may be different. Use in other age groups, including children and young adults, could be investigated. SPARX is likely to be a cheaper way of delivering treatment than usual treatment, although this needs to be established by a formal cost-benefit analysis. The development of an adjunctive clinical monitoring tool to allow clinicians to track progress of their patients would be worthwhile.

\section{We thank Maru Nihoniho and staff from Metia Interactive for their} invaluable work developing SPARX; the young people who helped to design SPARX; the young people who participated in the study and provided us with useful feedback on their experiences of SPARX; the clinicians and school guidance counsellors who recruited participants for the study; the research assistants who assisted with data collection across all the study sites; the local investigators who assisted with individual site coordination and data collection; the Cultural Advisory Group who provided valuable feedback and guidance to the research team in the course of the study; Kaumātua Rawiri Wharemate for his ongoing support for the Werry Centre and this study in particular;

Candace Bagnall for all the work she has done to help people access effective mental health services in New Zealand; Tiki Taane for letting us use the track "Faded" in SPARX for the duration of the trial; and lona Bailey, a general practitioner in Tauranga, who suggested computerising treatment for adolescent depression to associate SNM a decade ago. The trial protocol is available on www.sparx.org.nz.

Contributors: SNM conceived this study and is guarantor. SNM, KS, MS, CF, TF, and MFGL designed and initiated the study. SNM, KS, MS, and TF were responsible for participant recruitment, data gathering, and preparation of the database. MFGL assisted in data gathering. CF was responsible for statistical analysis. SNM monitored processes, with particular reference to managing adverse events. SNM wrote the first draft of the manuscript. KS, MS, CF, MFGL, and TF revised the manuscript. All authors contributed to and approved the final version of the paper. All authors had full access to all of the data and can take responsibility for its integrity. The research team has full access to all the data and the corresponding author had final responsibility for the decision to submit the paper.
Funding: The New Zealand Ministry of Health funded the study but had no direct involvement in the design or conduct of the study, analysis of the data, or writing up of the results.

Competing interests: All authors have completed the ICMJE uniform disclosure form at www.icmje.org/coi_disclosure.pdf (available on request from the corresponding author) and declare that: SNM, KS, MS, $\mathrm{CF}, \mathrm{TF}$, and MFGL were supported by the University of Auckland for the submitted work; SNM, KS, MS, CF, TF and MFGL have no relationships with the University of Auckland that might have an interest in the submitted work in the previous three years; and SNM, KS, MS, $\mathrm{CF}, \mathrm{TF}$, and MFGL have no non-financial interests that may be relevant to the submitted work.

Ethical approval: This study was approved by the multiregion ethics committee (New Zealand Ministry of Health) MEC/08/12/159. Participants aged 16 years and older provided their own informed consent to participate in the study. For participants 15 years and younger, parental/guardian informed consent was obtained together with the informed consent of the participant.

Data sharing: The dataset is available on request from the corresponding author at s.merry@auckland.ac.nz.

Lewinsohn PM, Rohde P. Seeley JR. Major depressive disorder in older adolescents: prevalence, risk factors and clinical implications. Clin Psychol Rev 1998;18:765-94.

2 World Health Organization. Global health risks: mortality and burden of disease attributable to selected major risks. WHO, 2009

3 Watanabe N, Hunot V, Omori IM, Churchill R, Furukawa TA. Psychotherapy for depression among children and adolescents: a systematic review. Acta Psychiatr Scand 2007;116:84-95

4 National Institute for Health and Clinical Excellence. Depression in children and young people: identification and management in primary, community and secondary care. NICE, 2005.

5 Kataoka S, Zhang L, Wells K. Unmet need for mental health care among U.S. children: variations by ethnicity and insurance status. Am J Psychiatry 2002;159:1548-55.

6 Mariu KR, Merry SN, Robinson EM, Watson PD. Seeking professional help for mental health problems, among New Zealand secondary school students. Clin Child Psychol Psychiatry 2011:1-14.

7 Layard R, Bell SB, Clarke D. The depression report. A new deal for depression and anxiety disorders. London School of Economics, 2006.

8 Le Surf A, Lynch G. Exploring young people's perception relevant to counselling: a qualitative study. Br J Guid Counc 1999;27:231-43.

9 Prensky M. Digital natives, digital immigrants part 1. On the Horizon 2001;9:1-6.

10 McCrone P, Knapp M, Proudfoot J, Ryden C, Cavanagh K, Shapiro DA, et al. Cost-effectiveness of computerised cognitive-behavioural therapy for anxiety and depression in primary care: randomised controlled trial. Br J Psychiatry 2004;185:55-62.

11 Andrews G, Cuijpers P, Craske MG, McEvoy P, Titov N. Computer therapy for the anxiety and depressive disorders is effective, acceptable and practical health care: a meta-analysis. PLOS One 2010;5:1-6.

12 Christensen H, Griffiths KM, Farrer L. Adherence in internet interventions for anxiety and depression. J Med Internet Res 2009;11:e13.

13 Richardson T, Stallard P, Velleman S. Computerised cognitive behavioural therapy for the prevention and treatment of depression and anxiety in children and adolescents: a systematic review. Clin Child Fam Psychol Rev 2010;13:275-90.

14 Calear AL, Christensen $\mathrm{H}$. Review of internet-based prevention and treatment programs for anxiety and depression in children and adolescents. Med J Aust 2010;192:S12-4.

15 Stasiak K. Computer-administered cognitive behavioural self-help intervention for adolescents with mild to moderate depressive symptoms: programme development and examination of feasibility, efficacy and acceptability. Unpublished PhD Thesis: University of Auckland, 2008.

16 Fleming T, Dixon R, Frampton C, Merry S. A pragmatic randomized controlled trial of computerized CBT (SPARX) for symptoms of depression among adolescents excluded from mainstream education. Behav Cogn Psychother 2011; published online 5 Dec.

17 Stallard P, Richardson T, Velleman S, Attwood M. Computerized CBT (Think, Feel, Do) for depression and anxiety in children and adolescents: outcomes and feedback from a pilot randomized controlled trial. Behav Cogn Psychother 2011;39:273-84.

18 Poznanski EO, Mokros HB. Manual for the Children's Depression Rating Scale-Revised. Western Psychological Services, 1996.

19 Kroenke K, Spitzer RL, Williams JB. The PHQ-9: validity of a brief depression severity measure. J Gen Intern Med 2001;16:606-13.

20 Brooks SJ, Kutcher S. Diagnosis and measurement of adolescent depression: a review of commonly utilized instruments. J Child Adolesc Psychopharmacol 2001;11:341-76.

21 Myers K, Winters NC. Ten-year review of rating scales. II: scales for internalizing disorders. J Am Acad Child Adolesc Psychiatry 2002;41:634-59.

22 Emslie GJ, Rush J, Weinberg WA, Kowatch RA, Hughes CW, Carmody T, et al. A double-blind, randomized, placebo-controlled trial of fluoxetine in children and adolescents with depression. Arch Gen Psychiatry 1997;54:1031-7.

23 Reynolds WM. Reynolds Adolescent Depression Scale, 2nd Ed (RADS-2): Professional Manual. Psychological Assessment Resources, 2002.

24 Walker L, Merry S, Watson PD, Robinson E, Crengle S, Schaaf D. The Reynolds adolescent depression scale in New Zealand adolescents. Aust N Z J Psychiatry 2005;39:136-40.

25 Angold A, Costello EJ, Messer SC, Pickles A, Winder F, Silver D. The development of a short questionnaire for use in epidemiological studies of depression in children and adolescents. Int J Methods Psychiatr Res 1995;5:237-49. 


\section{What is already known on this topic}

Although depression is common in adolescents, most with depressive symptoms do not obtain professional help Cognitive behavioural therapy is an effective intervention, but services find it difficult to deliver psychosocial therapies Computerised cognitive behavioural therapy is effective for adults with depression, but little is known about its effect in adolescents

\section{What this study adds}

A specific computerised cognitive behavioural therapy resource (SPARX) for adolescents with depressive symptoms was as effective a treatment as usual care in primary care settings in New Zealand

Adolescents enjoyed SPARX and adherence to the program was high

SPARX is a promising treatment for adolescents with depressive symptoms and has the potential to increase substantially access to care in primary care settings

26 Endicott J, Nee J, Yang R, Wohlberg C. Pediatric Quality of Life Enjoyment and Satisfaction Questionnaire (PQ-LES-Q): reliability and validity. J Am Acad Child Adolesc Psychiatry 2006;45:401-7.

27 Spence SH. A measure of anxiety symptoms among children. Behav Res Ther 1998;36:545-66.

28 Kazdin AE, French NH, Unis AS, Esveldt-Dawson K, Sherick RB. Hopelessness, depression and suicidal intent among psychiatrically disturbed inpatient children. $J$ Consult Clin Psychol 1983;51:504-10.

29 Guy W. ECDEU assessment manual for psychopharmacology. Government Printing Office, 1976.

30 Piaggio G, Elbourne DR, Altman DG, Pocock SJ, Evans SJW. Reporting of noninferiority and equivalence randomized trials: an extension of the CONSORT statement. JAMA 2006;295:1152-60

31 March JS, Vitiello B. Clinical messages from the treatment for adolescents with depression study (TADS). Am J Psychiatry 2009;166:1118-23

32 Hetrick S, Merry S, McKenzie J, Sindahl P, Proctor M. Selective serotonin reuptake inhibitors (SSRIs) for depression in children and adolescents. Cochrane Database Syst Rev 2007(3):CD004851.

33 Gledhill J, Garralda M. The short-term outcome of depressive disorder in adolescents attending primary care: a cohort study. Soc Psychiatry Psychiatr Epidemiol 2011:46:993-1002.

34 New Zealand Guidelines Group. Identification of common mental disorders and management of depression in primary care. An evidence-based best practice guideline. New Zealand Guidelines Group, 2008.
35 Lewinsohn PM, Clarke GN, Seeley JR. Major depression in community adolescents: age at onset, episode duration, and time of recurrence. J Am Acad Child Adolesc Psychiatry 1994:33:809-18.

36 Calear AL, Christensen H, Mackinnon A, Griffiths KM, O'Kearney R. The YouthMood project: a cluster randomized controlled trial of an online cognitive behavioral program with adolescents. J Consult Clin Psychol 2009;77:1021-32.

37 Shandley K, Austin D, Klein B, Kyrios M. An evaluation of 'Reach Out Central': an online gaming program for supporting the mental health of young people. Health Educ Res 2010;25:563-74.

38 Proudfoot J, Goldberg D, Mann A, Everitt B, Marks I, Gray JA. Computerized, interactive, multimedia cognitive-behavioural program for anxiety and depression in general practice. Psychol Med 2003;33:217-27.

\section{Accepted: 9 March 2012}

\section{Cite this as: BMJ 2012:344:e2598}

This is an open-access article distributed under the terms of the Creative Commons Attribution Non-commercial License, which permits use, distribution, and reproduction in any medium, provided the original work is properly cited, the use is non commercial and is otherwise in compliance with the license. See: http://creativecommons.org/licenses/by$\mathrm{nc} / 2.0 /$ and http://creativecommons.org/licenses/by-nc/2.0/legalcode. 


\section{Tables}

Table 1/ Personal characteristics of help seeking adolescents with depression allocated to a computerised cognitive behavioural therapy intervention (SPARX) or to treatment as usual. Values are percentages (numbers) unless stated otherwise

\begin{tabular}{|c|c|c|}
\hline Characteristics & SPARX group $(n=94)$ & Treatment as usual group $(n=93)$ \\
\hline Female & $62.8(59)$ & $68.8(64)$ \\
\hline Male & $37.2(35)$ & $31.2(29)$ \\
\hline \multicolumn{3}{|l|}{ Age (years): } \\
\hline Mean (SD) age & $15.55(1.54)$ & $15.58(1.66)$ \\
\hline$\leq 14$ & $41.5(39)$ & $37.6(35)$ \\
\hline $15-16$ & $40.4(38)$ & $40.9(38)$ \\
\hline$\geq 17$ & $18.1(17)$ & $21.5(20)$ \\
\hline \multicolumn{3}{|l|}{ Ethnicity: } \\
\hline New Zealand European & $58.5(55)$ & $60.2(56)$ \\
\hline Māori & $25.5(24)$ & $22.6(21)$ \\
\hline Pacific people & $8.5(8)$ & $7.5(7)$ \\
\hline Asian* ${ }^{*}$ & $4.3(4)$ & $8.6(8)$ \\
\hline Other & $3.2(3)$ & $1.1(1)$ \\
\hline \multicolumn{3}{|l|}{ Setting: } \\
\hline Schools & $54.3(51)$ & $55.9(52)$ \\
\hline Non-schools & $45.7(43)$ & $44.1(41)$ \\
\hline
\end{tabular}

${ }^{*}$ Chinese, Japanese, Korean, Indian, Malaysian, Fijian Indian, Cambodian, and Thai. 


\begin{tabular}{|c|c|c|c|c|c|c|}
\hline \multirow[b]{2}{*}{ Measure } & \multicolumn{3}{|c|}{ Mean (SD) SPARX* group } & \multicolumn{3}{|c|}{ Mean (SD) treatment as usual group } \\
\hline & Baseline & Post-intervention & 3 month follow-up & Baseline & Post-intervention & 3 month follow-up \\
\hline \multicolumn{7}{|c|}{ Primary outcome } \\
\hline \multicolumn{7}{|l|}{ CDRS-R: } \\
\hline PP $n=143$ & $44.28(11.13)$ & $34.14(11.93)$ & $29.47(9.70)$ & $43.78(9.67)$ & $36.71(11.05)$ & $30.75(8.73)$ \\
\hline ITT $n=187$ & $43.02(11.12)$ & $33.92(11.19)$ & $28.96(9.11)$ & $42.09(10.38)$ & $35.07(9.71)$ & $29.37(9.87)$ \\
\hline \multicolumn{7}{|c|}{ Secondary outcomes } \\
\hline \multicolumn{7}{|l|}{ RADS-2: } \\
\hline $\mathrm{PP} n=143$ & $76.16(13.35)$ & $65.44(14.89)$ & $60.87(15.01)$ & $77.99(13.46)$ & $70.21(13.43)$ & $62.73(14.84)$ \\
\hline ITT $n=187$ & $74.83(13.35)$ & $65.19(14.52)$ & $60.68(12.51)$ & $75.52(14.42)$ & $68.24(12.31)$ & $61.45(14.68)$ \\
\hline \multicolumn{7}{|l|}{ MFQ: } \\
\hline $\mathrm{PP} n=143$ & $28.73(11.68)$ & $19.02(12.56)$ & $15.97(10.84)$ & $32.25(11.48)$ & $24.74(11.89)$ & $19.27(11.46)$ \\
\hline ITT $n=187$ & $27.59(12.15)$ & $19.10(12.29)$ & $15.77(10.02)$ & $29.41(12.99)$ & $22.96(11.87)$ & $17.92(11.88)$ \\
\hline \multicolumn{7}{|l|}{ PQ-LES-Q: } \\
\hline $\mathrm{PP} n=143$ & $27.78(9.40)$ & $33.17(10.39)$ & $34.99(10.04)$ & $26.39(8.49)$ & $29.93(9.23)$ & $33.79(8.75)$ \\
\hline ITT n=187 & $28.35(9.24)$ & $33.29(8.92)$ & 35.14 (8.32) & $28.13(9.85)$ & $31.26(7.89)$ & $34.87(8.93)$ \\
\hline \multicolumn{7}{|c|}{ Kazdin HPLS: } \\
\hline $\mathrm{PP} n=143$ & $6.41(4.15)$ & $4.09(3.78)$ & $3.43(3.30)$ & $6.61(4.29)$ & $5.40(4.32)$ & $3.74(3.56)$ \\
\hline ITT $n=187$ & $6.17(3.98)$ & $4.31(4.00)$ & $3.61(2.75)$ & $6.15(4.32)$ & $4.99(3.93)$ & 3.54 (3.52) \\
\hline \multicolumn{7}{|l|}{ SCAS (total) } \\
\hline $\mathrm{PP} n=143$ & $39.19(16.03)$ & $29.91(15.24)$ & $25.96(15.28)$ & $38.53(15.69)$ & 32.55 (14.99) & $27.98(15.25)$ \\
\hline ITT $n=187$ & $37.74(16.44)$ & $28.86(12.23)$ & $25.60(10.32)$ & $35.36(16.30)$ & $29.97(12.23)$ & $26.25(11.28)$ \\
\hline \multicolumn{7}{|c|}{ Separation anxiety: } \\
\hline PP $n=143$ & $4.07(2.55)$ & $3.07(2.46)$ & $2.31(2.17)$ & $4.54(3.37)$ & $3.90(3.34)$ & $3.18(2.80)$ \\
\hline ITT $n=187$ & $3.79(2.56)$ & $2.91(2.15)$ & $2.25(2.24)$ & $4.15(3.17)$ & $3.66(2.95)$ & $3.00(2.37)$ \\
\hline \multicolumn{7}{|c|}{ Social phobia: } \\
\hline $\mathrm{PP} n=143$ & $9.16(3.66)$ & $7.56(3.58)$ & $6.96(3.66)$ & 8.67 (3.37) & 7.40 (3.51) & 7.01 (3.57) \\
\hline ITT n=187 & $8.63(3.88)$ & $7.27(3.64)$ & $6.72(2.71)$ & $8.03(3.55)$ & $6.67(3.03)$ & $6.57(2.83)$ \\
\hline \multicolumn{7}{|c|}{$\begin{array}{l}\text { Obsessive-compulsive } \\
\text { disorder: }\end{array}$} \\
\hline $\mathrm{PP} n=143$ & $6.32(3.63)$ & $4.56(3.42)$ & $3.39(3.21)$ & $6.53(4.01)$ & $5.55(3.82)$ & $4.09(3.86)$ \\
\hline ITT $n=187$ & $6.46(3.71)$ & $4.61(3.24)$ & $3.66(2.63)$ & $5.92(4.17)$ & $5.17(3.21)$ & $3.77(2.71)$ \\
\hline \multicolumn{7}{|c|}{ Panic/agoraphobia: } \\
\hline $\mathrm{PP} n=143$ & $6.77(5.15)$ & $4.58(4.65)$ & $3.86(4.04)$ & $6.07(4.62)$ & $4.63(3.80)$ & $4.00(3.87)$ \\
\hline ITT $n=187$ & $6.45(5.09)$ & $4.40(3.65)$ & $3.82(2.93)$ & $5.44(4.55)$ & $4.08(3.69)$ & $3.52(3.27)$ \\
\hline \multicolumn{7}{|c|}{ Physical injury fears: } \\
\hline $\mathrm{PP} n=143$ & $4.61(2.93)$ & $3.98(2.89)$ & $3.85(2.85)$ & $4.32(3.27)$ & $3.82(3.04)$ & $3.78(2.69)$ \\
\hline ITT $n=187$ & $4.43(3.11)$ & $3.73(1.94)$ & $3.76(1.79)$ & $4.10(3.27)$ & $3.61(1.90)$ & $3.62(1.97)$ \\
\hline \multicolumn{7}{|c|}{ Generalised anxiety: } \\
\hline $\mathrm{PP} n=143$ & $8.18(3.39)$ & $6.12(3.39)$ & $5.51(3.52)$ & $8.39(3.25)$ & $7.41(2.94)$ & $5.89(2.94)$ \\
\hline ITT $n=187$ & $7.92(3.57)$ & $6.01(2.96)$ & $5.47(2.89)$ & $7.69(3.43)$ & $6.61(3.04)$ & $5.51(2.81)$ \\
\hline
\end{tabular}

CDRS-R=children's depression rating scale-revised; $\mathrm{PP}=$ per protocol population; ITT=intention to treat population; RADS-2=Reynolds adolescent depression scale-second edition; MFQ=mood and feelings questionnaire; PQ-LES-Q=pediatric quality of life enjoyment and satisfaction questionnaire; Kazdin HPLS=Kazdin hopelessness scale for children; SCAS=Spence children's anxiety scale.

For intention to treat population the means and standard deviations at post-intervention and at three month follow-up include individual imputed estimates. ${ }^{*}$ Computerised cognitive behavioural therapy intervention. 
Table 3| Mean improvements and mean difference in change scores from baseline to post-intervention and from post-intervention to three month follow-up

\begin{tabular}{|c|c|c|c|c|}
\hline \multirow[b]{2}{*}{ Measure } & \multicolumn{2}{|c|}{ Mean change $(95 \% \mathrm{Cl})$ post-intervention } & \multirow{2}{*}{$\begin{array}{l}\text { Mean difference in change scores } \\
\qquad(95 \% \mathrm{Cl})\end{array}$} & \multirow[b]{2}{*}{$P$ value } \\
\hline & SPARX $^{*}$ group & Treatment as usual group & & \\
\hline \multicolumn{5}{|c|}{ Baseline to post-intervention } \\
\hline \multicolumn{5}{|c|}{ Primary outcome: CDRS-R: } \\
\hline $\mathrm{PP} n=143$ & $10.32(8.15$ to 12.48$)$ & 7.59 (5.43 to 9.75$)$ & $2.73(-0.31$ to 5.77$)$ & 0.079 \\
\hline ITT $n=187$ & 9.05 (7.07 to 11.03$)$ & 7.45 (5.46 to 9.45$)$ & $1.60(-1.21$ to 4.41$)$ & 0.264 \\
\hline \multicolumn{5}{|c|}{ Secondary outcomes: } \\
\hline \multicolumn{5}{|c|}{ RADS-2: } \\
\hline PP $n=143$ & $11.20(8.50$ to 13.91$)$ & 7.55 (4.86 to 10.25$)$ & $3.65(-0.15$ to 7.45$)$ & 0.060 \\
\hline ITT $n=187$ & 9.78 (7.12 to 12.43$)$ & 7.16 (4.54 to 9.79$)$ & $2.62(-1.04$ to 6.27$)$ & 0.161 \\
\hline \multicolumn{5}{|l|}{ MFQ: } \\
\hline $\mathrm{PP} n=143$ & 10.64 (8.22 to 13.06$)$ & $6.90(4.49$ to 9.31$)$ & $3.74(0.33$ to 7.16$)$ & 0.032 \\
\hline ITT $n=187$ & $8.90(6.56$ to 11.24$)$ & $6.08(3.60$ to 8.57$)$ & $2.81(-0.72$ to 6.35$)$ & 0.118 \\
\hline \multicolumn{5}{|l|}{ PQ-LES-Q: } \\
\hline $\mathrm{PP} n=143$ & 5.71 (3.95 to 7.47$)$ & 3.47 (1.72 to 5.23$)$ & $2.23(-0.24$ to 4.71$)$ & 0.077 \\
\hline ITT $n=187$ & 5.01 (3.40 to 6.63 ) & 3.14 (1.43 to 4.85$)$ & $1.87(-0.51$ to 4.26$)$ & 0.124 \\
\hline \multicolumn{5}{|c|}{ Kazdin HPLS: } \\
\hline$P P n=143$ & 2.37 (1.57 to 3.18$)$ & $1.16(0.36$ to 1.96$)$ & $1.22(0.09$ to 2.35$)$ & 0.035 \\
\hline ITT $n=187$ & $1.84(1.07$ to 2.62$)$ & $1.15(0.41$ to 1.89$)$ & $0.69(-0.41$ to 1.79$)$ & 0.218 \\
\hline \multicolumn{5}{|c|}{ SCAS (total): } \\
\hline $\mathrm{PP} n=143$ & $9.18(6.76$ to 11.60$)$ & 6.09 (3.67 to 8.50$)$ & $3.09(-0.31$ to 6.50$)$ & 0.075 \\
\hline ITT $n=187$ & $8.50(6.05$ to 10.94$)$ & 5.69 (3.28 to 8.11$)$ & $2.80(-0.53$ to 6.13$)$ & 0.099 \\
\hline \multicolumn{5}{|c|}{ Separation anxiety: } \\
\hline $\mathrm{PP} n=143$ & $1.10(0.60$ to 1.61$)$ & 0.58 (0.08 to 1.09$)$ & $0.52(-0.19$ to 1.23$)$ & 0.153 \\
\hline ITT $n=187$ & 0.94 (0.44 to 1.44$)$ & $0.43(-0.12$ to 0.99$)$ & $0.51(-0.22$ to 1.23$)$ & 0.170 \\
\hline \multicolumn{5}{|c|}{ Social phobia: } \\
\hline $\mathrm{PP} n=143$ & $1.51(0.85$ to 2.16$)$ & 1.37 (0.72 to 2.02$)$ & $0.14(-0.78$ to 1.05$)$ & 0.773 \\
\hline ITT $n=187$ & $1.25(0.64$ to 1.87$)$ & 1.51 (0.87 to 2.15$)$ & $-0.26(-1.14$ to 0.62$)$ & 0.566 \\
\hline \multicolumn{5}{|c|}{ Obsessive-compulsive disorder: } \\
\hline$P P n=143$ & 1.79 (1.12 to 2.45$)$ & $0.91(0.25$ to 1.57$)$ & $0.88(-0.05$ to 1.81$)$ & 0.065 \\
\hline ITT $n=187$ & $1.72(1.14$ to 2.30$)$ & $0.82(0.25$ to 1.40$)$ & $0.89(0.09$ to 1.70$)$ & 0.030 \\
\hline \multicolumn{5}{|c|}{ Panic/agoraphobia: } \\
\hline $\mathrm{PP} n=143$ & 2.06 (1.36 to 2.77$)$ & 1.59 (0.89 to 2.30$)$ & $0.47(-0.52$ to 1.47$)$ & 0.352 \\
\hline ITT $n=187$ & 1.84 (1.19 to 2.50$)$ & 1.56 (0.88 to 2.23$)$ & $0.29(-0.67$ to 1.24$)$ & 0.553 \\
\hline \multicolumn{5}{|c|}{ Physical injury fears: } \\
\hline $\mathrm{PP} n=143$ & $0.61(0.22$ to 1.00$)$ & $0.55(0.16$ to 0.94$)$ & $0.06(-0.49$ to 0.61$)$ & 0.475 \\
\hline ITT $n=187$ & 0.65 (0.29 to 1.02$)$ & $0.53(0.15$ to 0.90$)$ & $0.13(-0.39$ to 0.65$)$ & 0.632 \\
\hline \multicolumn{5}{|c|}{ Generalised anxiety: } \\
\hline $\mathrm{PP} n=143$ & 2.09 (1.48 to 2.69$)$ & $1.18(0.58$ to 1.79$)$ & $0.91(0.06$ to 1.75$)$ & 0.037 \\
\hline ITT $n=187$ & $1.84(1.27$ to 2.41$)$ & $1.11(0.55$ to 1.67$)$ & $0.73(-0.06$ to 1.52$)$ & 0.069 \\
\hline \multicolumn{5}{|c|}{ Post-intervention to three month follow-up } \\
\hline \multicolumn{5}{|c|}{ Primary outcome: CDRS-R: } \\
\hline $\mathrm{PP} n=143$ & $4.68(2.50$ to 6.86$)$ & 5.95 (3.79 to 8.12$)$ & $-1.28(-4.35$ to 1.79$)$ & 0.412 \\
\hline ITT $n=187$ & $4.96(2.87$ to 7.05$)$ & $5.70(3.65$ to 7.76$)$ & $-0.75(-3.61$ to 2.12$)$ & 0.609 \\
\hline \multicolumn{5}{|c|}{ Secondary outcomes } \\
\hline \multicolumn{5}{|l|}{ RADS-2: } \\
\hline $\mathrm{PP} n=143$ & 4.57 (1.58 to 7.59$)$ & $7.48(4.48$ to 10.48$)$ & $-2.91(-7.17$ to 1.34$)$ & 0.178 \\
\hline ITT $n=187$ & 4.51 (1.55 to 7.47$)$ & 6.79 (3.91 to 9.66$)$ & $-2.28(-6.41$ to 1.86$)$ & 0.280 \\
\hline
\end{tabular}


Table 3 (continued)

\begin{tabular}{|c|c|c|c|c|}
\hline \multirow[b]{2}{*}{ Measure } & \multicolumn{2}{|c|}{ Mean change $(95 \% \mathrm{Cl})$ post-intervention } & \multirow{2}{*}{$\begin{array}{l}\text { Mean difference in change scores } \\
\qquad(95 \% \mathrm{Cl})\end{array}$} & \multirow[b]{2}{*}{$P$ value } \\
\hline & SPARX $^{\star}$ group & Treatment as usual group & & \\
\hline $\mathrm{PP} n=143$ & 3.04 (0.53 to 5.56$)$ & 5.47 (2.96 to 7.96$)$ & $-2.42(-5.97$ to 1.12$)$ & 0.179 \\
\hline ITT n=187 & $3.33(0.99$ to 5.67$)$ & 5.04 (2.74 to 7.35$)$ & $-1.71(-5.05$ to 1.62$)$ & 0.314 \\
\hline \multicolumn{5}{|l|}{ PQ-LES-Q: } \\
\hline $\mathrm{PP} n=143$ & $1.82(0.16$ to 3.80$)$ & 3.85 (1.88 to 5.81$)$ & $-2.03(-4.82$ to 0.76$)$ & 0.152 \\
\hline ITT n=187 & $1.85(0.02$ to 3.68$)$ & $3.62(1.80$ to 5.43$)$ & $-1.77(-0.81$ to 4.34$)$ & 0.179 \\
\hline \multicolumn{5}{|c|}{ Kazdin HPLS: } \\
\hline$P P n=143$ & $0.66(0.10$ to 1.41$)$ & 1.67 (0.92 to 2.42$)$ & $-1.01(-2.08$ to 0.06$)$ & 0.063 \\
\hline ITT n=187 & $0.70(0.03$ to 1.38$)$ & $1.46(0.76$ to 2.15$)$ & $-0.75(-1.68$ to 0.18$)$ & 0.112 \\
\hline \multicolumn{5}{|c|}{ SCAS (total): } \\
\hline$P P n=143$ & 3.95 (1.48 to 6.42$)$ & 4.57 (2.12 to 7.02$)$ & $-0.62(-4.10$ to 2.86$)$ & 0.726 \\
\hline ITT $n=187$ & 3.26 (1.01 to 5.51$)$ & $3.72(1.40$ to 6.05$)$ & $-0.46(-3.68$ to 2.76$)$ & 0.778 \\
\hline \multicolumn{5}{|c|}{ Separation anxiety: } \\
\hline$P P n=143$ & $0.76(0.23$ to 1.30$)$ & $0.72(0.19$ to 1.25$)$ & $0.04(-0.71$ to 0.80$)$ & 0.914 \\
\hline ITT $n=187$ & 0.66 (0.19 to 1.13$)$ & $0.66(0.15$ to 1.17$)$ & $0(-0.68$ to 0.69$)$ & 0.998 \\
\hline \multicolumn{5}{|c|}{ Social phobia: } \\
\hline$P P n=143$ & 0.61 (0.02 to 1.23$)$ & $0.40(0.22$ to 1.02$)$ & $0.21(-0.67$ to 1.09$)$ & 0.640 \\
\hline ITT n=187 & $0.55(-0.05$ to 1.15$)$ & $0.10(-0.48$ to 0.68$)$ & $0.44(-0.40$ to 1.28$)$ & 0.299 \\
\hline \multicolumn{5}{|c|}{ Obsessive-compulsive disorder: } \\
\hline$P P n=143$ & $1.17(0.54$ to 1.80$)$ & $1.46(0.84$ to 2.09$)$ & $-0.29(-1.18$ to 0.60$)$ & 0.516 \\
\hline ITT $n=187$ & $0.95(0.39$ to 1.51$)$ & $1.40(0.83$ to 1.97$)$ & $-0.45(-1.25$ to 0.35$)$ & 0.268 \\
\hline \multicolumn{5}{|c|}{ Panic/agoraphobia: } \\
\hline $\mathrm{PP} n=143$ & $0.72(0.02$ to 1.46$)$ & $0.63(0.10$ to 1.36$)$ & $0.09(-0.95$ to 1.13$)$ & 0.867 \\
\hline ITT n=187 & $0.58(-0.09$ to 1.25$)$ & $0.57(-0.09$ to 1.22$)$ & $0.02(-0.94$ to 0.97$)$ & 0.975 \\
\hline \multicolumn{5}{|c|}{ Physical injury fears: } \\
\hline$P P \mathrm{n}=143$ & $0.13(-0.32$ to 0.58$)$ & $0.04(-0.40$ to 0.48$)$ & $0.09(-0.54$ to 0.72$)$ & 0.781 \\
\hline ITT $n=187$ & $0.03(-0.44$ to 0.37$)$ & $0.01(-0.41$ to 0.40$)$ & $0.02(-0.59$ to 0.55$)$ & 0.934 \\
\hline \multicolumn{5}{|c|}{ Generalised anxiety: } \\
\hline$P P \mathrm{n}=143$ & 0.61 (0.04 to 1.27$)$ & $1.26(0.61$ to 1.90$)$ & $-0.64(-1.56$ to 0.28$)$ & 0.170 \\
\hline ITT n=187 & $0.54(-0.08$ to 1.16$)$ & $1.10(0.49$ to 1.70$)$ & $-0.56(-1.42$ to 0.31$)$ & 0.209 \\
\hline
\end{tabular}

$\mathrm{PP}=$ per protocol population; ITT=intention to treat population.

Means and confidence intervals are derived from the linear mixed modelling analysis. Positive values favour SPARX (negative values favour treatment as usual).

*Computerised cognitive behavioural therapy intervention. 
Table 4| Depression severity at entry and mean changes on children's depression rating scale-revised by intervention

\begin{tabular}{lcc} 
& & Mean change $(95 \%$ Cl) \\
\cline { 2 - 3 } Severity of depression & & Treatment as usual group \\
Possible $(n=103)$ & SPARX group & $2.94(0.45$ to 5.43$)$ \\
\hline Likely $(n=62)$ & $5.61(3.06$ to 8.16$)$ & $12.90(9.49$ to 16.32$)$ \\
\hline Very likely $(n=22)$ & $10.60(7.46$ to 13.74$)$ & $12.86(7.32$ to 18.40$)$
\end{tabular}

${ }^{*}$ Children's depression rating scale-revised raw scores: $30-42$ (it is possible that a depressive disorder might be confirmed in a comprehensive diagnostic evaluation), 43-57 inclusive (a depressive disorder is likely to be confirmed in a comprehensive diagnostic evaluation), and 58-72 inclusive (a depressive disorder is very likely to be confirmed). ${ }^{7}$

†Computerised cognitive behavioural therapy intervention. 
Table 5| Adverse events recorded during study

\begin{tabular}{|c|c|c|}
\hline Variables & SPARX $^{\star}$ group & Treatment as usual group \\
\hline No of adverse events & 28 & 21 \\
\hline \multicolumn{3}{|c|}{ Relation to study participation: } \\
\hline Unrelated & 17 & 10 \\
\hline Possible & 11 & 10 \\
\hline Probable & 0 & 1 \\
\hline \multicolumn{3}{|l|}{ Outcome: } \\
\hline Continue with study & 26 & 20 \\
\hline Withdrawn from study & 2 & 1 \\
\hline
\end{tabular}




\section{Figures}

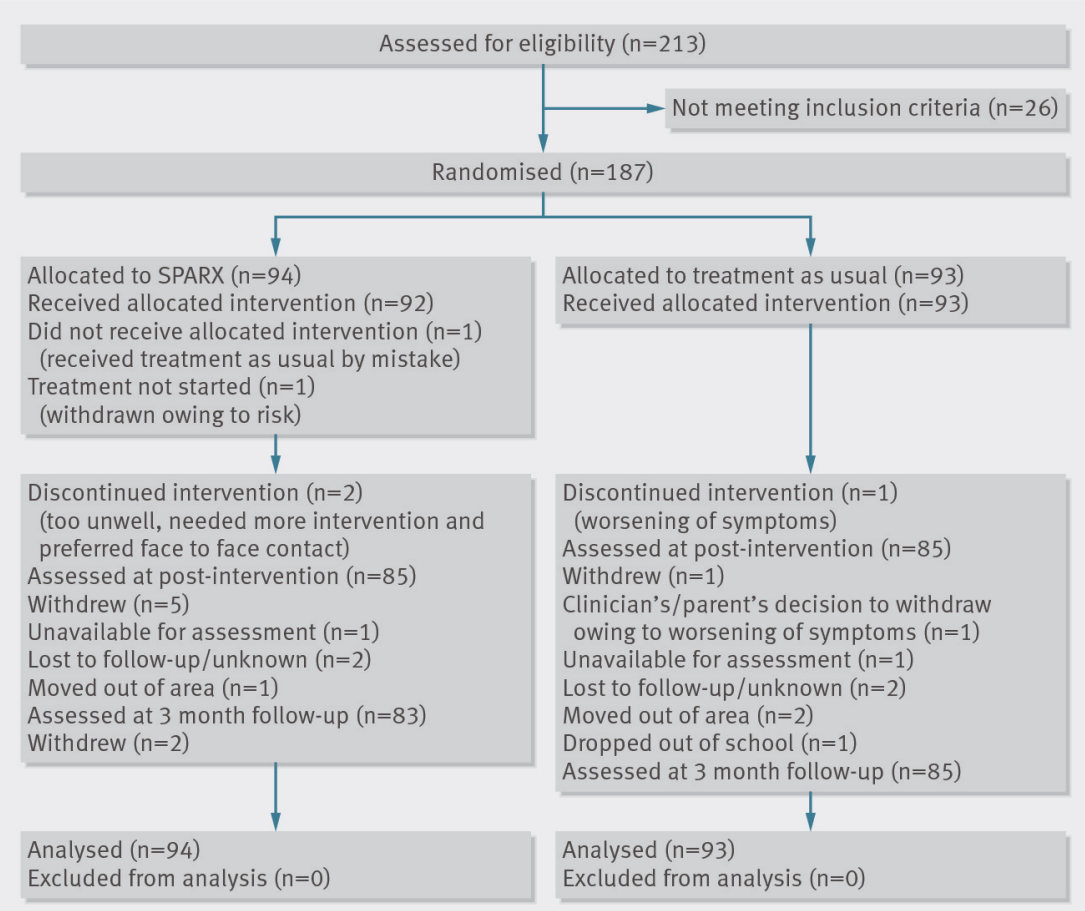

Fig 1 Flow of participants through study

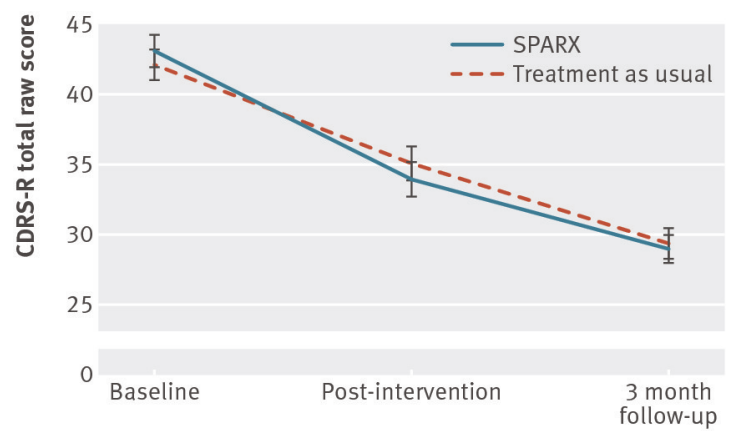

Fig 2 Changes (standard errors) on children's depression rating scale-revised (CDRS-R) 